\title{
CRITERIA FOR THE COMPLEXITY OF SUCCESSIVE ASTRONOMICAL PARADIGMS
}

\author{
${ }^{1}$ Panagiotis Papaspirou, ${ }^{2}$ Kostas Karamanos and ${ }^{3}$ Xenophon Moussas \\ ${ }^{1}$ Department of Physics, Section of Astrophysics, Astronomy and Mechanics, \\ National and Kapodistrian University of Athens, Panepistimiopoli, GR 15783 Zographos, Athens, Greece \\ ${ }^{2}$ Institute of Nuclear and Particle Physics, Complex Systems Group, \\ NCSR Demokritos, Aghia Paraskevi, Attiki, GR-15310, Athens, Greece \\ ${ }^{3}$ Department of Physics, Director of Astrophysics Laboratory, \\ National and Kapodistrian University of Athens, Panepistimiopoli, GR 15783 Zographos, Athens, Greece
}

Received 2014-02-05; Revised 2014-03-17; Accepted 2014-03-28

\begin{abstract}
We investigate the complexity of three successive astronomical paradigms in the science of Physics, namely the Ptolemaic paradigm, the Copernican paradigm and the Keplerian paradigm and mention briefly some characteristic facts about the colossal Newtonian paradigm. This complexity can be understood according to five criteria, as proposed by Thomas Kuhn, the father of the epistemological notion of the paradigm, as well as the founder of an important epistemological school within the realm of the 20th century. We propose that there does not exists an overall formal criterion for deciding among these rival paradigms, that is of the existing astronomical paradigms at the age Johannes Kepler formulated its own breakthrough within the science of Astronomy. The further evolution of the science of Astronomy, as well as the advent of the telescope era for investigating the celestial phenomena surely decided for the Newtonian paradigm, which can be understood as the epitome of all past astronomical and cosmological paradigms, yet the advance of the scientific study of the celestial phenomena did not evolved within a linear fashion, on the other hand, it has undergone many changes, subject to the great historical turns, that is the eras of the mentioned astronomical paradigms, during their evolution and their abandonment from the scientific community of the astronomers, the scholars and the polymaths of their age, respectively. We propose that each of Thomas Kuhn criteria imposes its own "complexity measure" of these paradigms, while the overall complexity criterion has to be regarded as the accumulating, overwhelming, empirical evidence, for finally deciding the new way of evolution and the novel turn within the science of Astronomy, especially in the post-Keplerian and surely in the post-Newtonian era.
\end{abstract}

Keywords: Paradigm, Astronomical Models, Cosmological Theories, Criteria of Paradigms, History of Astronomy

\section{INTRODUCTION}

The subject of paradigms in astronomy especially, but also in the realm of the physical sciences, as well as its introduction for the study of the social sciences, as a case study of the Kuhnian notion of the paradigm, is of great importance and has produced fruitful studies in the epistemological community (Kuhn, 1996; Gutting,
1980). In his famous paper "Objectivity, Value Judgment and Theory Choice", (Kuhn, 1977). Thomas Kuhn explores further the notion of the paradigm and proposes five criteria for the quality of the emerging and functioning paradigms within the realm of the physical sciences. Although the criteria do not form an exhaustive list, they are also not arbitrary, but surely belong to the working practice and experience of the working

(C) 2014 Panagiotis Papaspirou, Kostas Karamanos and Xenophon Moussas. This open access article is distributed under a Creative Commons Attribution (CC-BY) 3.0 license which permits unrestricted use, distribution, and reproduction in any medium, provided the original work is properly credited. DOI: 10.3844/pisp.2014.92.102 
theoretician and experimentalist. Kuhn proposes five normative categories for judging the strength and the scientific explorative ability of a paradigm, in general. These five criteria involve the accuracy, the internal and external consistency, the broad scope, the simplicity and the unification each paradigm introduces, as well as the fruitfulness it encloses.

We may explore even further the notions Kuhn introduces as the criteria of each paradigm. The criterion of accuracy refers to the fact that the consequences deducible from a paradigm have to be in demonstrated agreement with the results of existing experiments and observations. The link with the realm of inductive inference is straightforward and the working theoretician or experimentalist has to be ultimately confronted with experience, the empirical data play the role of the final test of each paradigm, although there can exist many rival theories, within a paradigm, or rival paradigms, which can explain, in their own terms, a corpus of available experimental or observational data.

The criterion of consistency refers to the fact that the paradigm has to be internally consistent, that is, that within this paradigm a proposition and the negation of this proposition cannot be proven at the same time, but also consistent with other accepted theories applicable to related aspects of nature. We may remark that the astronomical paradigms, up to the age of Johannes Kepler, are closely interconnected with other realms of discourse, such as Music theory (Marshall, 2009; Pacholczyk, 1996), Alchemy (Robertson, 2009; Hudson, 1992), or Astrology (Fisher, 2006), or other sciences, such as Medicine (Cooper, 2011) and Architecture. All these branches of knowledge form a tight semantic web and belong to, or form, the complete description of a closed, organically conceived universe, or Cosmos.

The criterion of broad scope refers to the fact that the consequences of the paradigm have to extend far beyond the particular observations, laws, or sub-theories, which had to be explained by the paradigm.

Another important criterion refers to the notion of simplicity, applicable to the structure and function of the paradigm, either in the sense of bringing order to the phenomena than in its absence would be individually isolated and, as a set, confused, but also in the sense of applying Occam's razor, that is on the economy and parsimony of the internal structure of the paradigm and its explanatory power. The criterion of fruitfulness refers to the fact that the paradigm should disclose new phenomena, or previously unnoted relationships among the already known facts (Kuhn, 1997; 2000).
We shall try to apply these criteria to the Ptolemaic, the Copernican and the Keplerian paradigm, as a multiset of competing astronomical paradigms, during the course of historical time, but also during the course of their "natural time" of evolution, shedding some light on the evolution of the physical theories, within the limited scope of the present investigation. All of these criteria could be understood as a kind of measure of the complexity each paradigm encloses, due to its structure, to its function and due to the modeling of the Universe it proposes and justifies (Margolis, 1993).

\section{THE STATUS OF THE ASTRONOMICAL PARADIGMS UP TO THE AGE OF KEPLER}

We shall try to offer a short picture of the status of astronomical paradigms, as they evolved up to the age of Johannes Kepler, that is up to the end of the pre-telescope era in the history of Astronomy (Dreyer, 1967). The age of Kepler can be considered as the transition from the era of Renaissance to the Baroque era, that is an age of social and cultural transformation, but also an age of interregnum, for the case of the physical sciences, that is a great quantum noospheric leap which eventually led to the post-newtonian era, the mechanization of the world-picture and the age of sailing (Dijksterhuis, 1986). Galileo Galilei introduces for the first time the usage of the telescope for astronomical observational purposes, an act which shall transform radically the science of Astronomy, offering a greater view on Cosmos, the ordered and harmonious universe, an autopoietic notion which has its origins in the Hellenic civilization and which spreads to the Byzantine, the Arabic and Islamic and eventually the European civilization (Laiou, 1992; Meri, 2004; Brotton, 2006).

The main cause of these astronomical paradigms is the correct reproduction and explanation of the planetary orbits, during their course within the passage of time, but also the formulation of a consistent cosmology, applicable to the then known universe. The universe of Ptolemy, Copernicus, Tycho Brahe, Kepler and Newton, in the general case, consisted of a set of simple justifiable facts, that is out of the presence of the Sun, the Earth, the Moon and the five visible by naked eye planets, that is of the wandering stars, together with the sphere of the fixed stars (Jones, 2010).

The planetary motions are observed via naked eye and indirectly, that is by their projection on the celestial sphere. These motions are complicated, that is their trajectory is complex, while the human curiosity and ingenuity tried to encompass them within sophisticated 
astronomical models and in turn, these models were imbedded within astronomical paradigms, which also served as cosmological paradigms, in the understanding of successive generations of scholars and polymaths, of astronomers and physicists belonging to successive great civilizations influenced by the Hellenistic and Alexandrian heritage (Taton and Wilson, 1989). The projection of the planetary orbits on the celestial sphere is thus the primary cause for the existence of the astronomical paradigms, while their birth strives from a very simple fact: The gyrations of the planets should be perfect circles, since the circle and the sphere can be regarded as the most perfect, that is the most symmetrical Euclidean geometrical figures. This fundamental concept already originates from the realm of the Hellenic scientific tradition and eventually becomes an Aristotelian dogma within the body of the Aristotelian physics (Llyod, 1973). This normative idea, a kind of meta-narrative principle, for the "book of nature which is written in the language of Mathematics", as Galileo stated, influenced indirectly by the Hellenistic and Alexandrian era, shall dominate the science of Astronomy for many centuries and shall turn to a metaphysical meta-principle, which every acceptable astronomical paradigm has to obey. Here, we shall encounter the genius of Kepler (Caspar, 1993), of the astronomer who ended a tradition lasting 1,400 years, from the age of Aristotle, by giving an arbitrary historical begin, as well as from the age of Claudius Ptolemy and up to his age.

By the detailed astronomical observational data we readily see that the planets deviate from their simple circular path, the path they should obey. A powerful sense of wonder emerges and the human mind takes its own path for discovering the true reasons, the true causes of the planetary motions, bridging Physics with Astronomy, Astronomy with Cosmology and Mathematics (Schroedinger, 1996).

At the very begin the core of each astronomical program, that is of every astronomical school, is to reproduce the complicated, that is the retrograde motion of the five planets, visible by naked eye, of the Sun, the Moon and of the Earth, by the strict usage of circular harmonic motions. This dictum refers to the Ptolemaic, the Copernican and the Keplerian astronomical model, while Newton integrates further Kepler's vision.

\section{THE OVERALL ASPECTS OF THE ASTRONOMICAL PARADIGMS}

Each paradigm offers many relevant faces of its entity, we may regard each paradigm as a kind of a polyhedron consisted of many faces, which interact with each other and form a holon, a mimetic structure, attributing to the paradigm its cohesion and its unity. Each paradigm offers a conceptual framework, attributes empirical laws to the physical reality, models in concrete ways the physical phenomena, imposes constraints on these, as well as to the acceptable error margins within each paradigm and is characterized by its historical evolution within the passage of time (Kuhn, 1996; 1977). These aspects can be classified according to the underlying mathematical structures of the paradigm, the empirical claims posed by it, the function of its theoretical terms, the role of the approximations undertaken and considered, the internal evolution of its structure and the inter-theoretic relations within its corpus (Laudan, 1986).

Of main importance are the existential quantifying terms of the paradigm and the extension of each of these paradigms, that is the numerical values obtained of a theoretical term from a given set of observational data. The values of the theoretical quantities can only be measured within a degree of imprecision and by the usage of auxiliary assumptions, which are plausible, but not certain. We observe that each paradigm encloses certain metaphysical assumptions and that it encloses physical reality as a whole, as a holistic interpretation of the physical world. Each paradigm can be considered not only as set of formal logical constructions, but also as a set of metaphors, by the combined usage of Geometry and Number theory, of observational facts and an interpretative Language and of a set of meta-principles, that is of laws and of constraints belonging to a kind of meta-language, a language to speak about the class of admissible paradigms of each historical period (Kuhn, 1974).

The scientific theories, as expressed within these paradigms, should have, as a map, or even better, a conceptual mapping, a one-to-one correspondence with natural reality. This epistemological thesis is considered to hold for each one of the fathers of the studied paradigms within the present article, both of Ptolemy, Copernicus, Kepler and Newton, held the view that their astronomical and cosmological models described accurately the physical reality, both on the ontological, as well as the theological status (Russo, 2004; Kuhn, 1957).

Also, we have to stress the fact that Theology and Epistemology serve as necessary ingredients of these paradigms, as understood within the context of the historical and social environment of the scientific community, composed by succeeding generations of 
astronomers, polymaths and philosophers, up to Kepler's and even Newton's era. The natural realism of these paradigms is always combined by theological and philosophical considerations, so that the notion of truth is referring in a direct manner with the physical laws and these are always referring to the divine status quo of the Universe, the reflection of the Nous of the Demiourge of Cosmos (Kutash, 2011). Within these paradigms, the combined effort of deductive and inductive, as well as the abductive reasoning, is present. Their role for the structure and the function for the succeeding astronomical paradigms, from the transition from the Ptolemaic to the Copernican and the Keplerian paradigm, serves always as a regulating factor within the era of their normal existence, the era of the normal science, as applied to these, but also in the periods of the scientific revolutions, of the eras of interregnum between these paradigms and the introduction of the novel paradigm (Heidelberger, 1976).

\section{THE KEPLERIAN PARADIGM}

We shall begin our investigation with the Keplerian paradigm, since this paradigm is a landmark between the successive astronomical paradigms we briefly study within this article. The Keplerian paradigm can be considered as a turning point within the history of Astronomy, of Physics and of Epistemology as well (Stephenson, 1994; Ben-Menahem, 2009a; 2009b).

Stated in a laconic fashion and according to the Keplerian paradigm, the Cosmos obeys the heliocentric principle, that is the Sun is positioned at the center of the universe.. Around the Sun the six planets revolve, that is Mercury, Venus, Earth, Mars, Jupiter and Saturn, while the Moon looses its stand as another revolving celestial body around the Earth and becomes Earth's satellite. The boundaries of the universe are formatted by the sphere of the fixed stars. The planetary orbits obey Kepler's three eponymous laws, that is the Law of areas, the Law of elliptic orbits and the Harmonic law (Frisch, 1858-1872). Each of these laws constitutes a great advance within the realm of the beforehand existing astronomical paradigms and brings in a dramatic fashion into the foreground the heliocentric doctrine, both in its pragmatic and physical dimension, as well as a revival of certain Neoplatonic and Neopythagorean teachings (Hopkins, 2001), although only the first mentioned component of Kepler's paradigm shall survive entirely in its concept in the Newtonian paradigm and in the evolution of the physical branch of Mechanics.
Moreover, Kepler poses for the first time a fundamental physical question: Is the Sun, or is the Earth the mover within the universe? He then proposes that the motive force of the solar system is located at the Sun, its effect diminishes with distance, so that Kepler introduces for the first time in the history of European astronomy the concept of a physical force, while at the same time visualizing this "gravitational" force with the aid of a conceptual scheme very akin to Faraday's lines of force, so that he can be considered as a forerunner of the concept of the physical field. Clearly, Kepler answers fundamental physical questions which arise from its newly formulated paradigm, in order to justify clearly his own heliocentric Cosmos, where the Earth is another moving planet around the Sun and where the terrestrial phenomena, that is the Physics applied on the surface of Earth, have to be considered and justified. Here, the geocentric and geostatic Ptolemaic paradigm stands as a clear rival, with respect to the explanation of the physical phenomena on Earth (Jones, 2010). The ingenuity of Kepler lies in the fact that he never diminishes the corpus of the Aristotelian physics, but he tries to complete it by newly discovered, by himself, laws and bold theoretical suggestions.

Kepler also uses two distinct meta-principles for shaping and structuring his universe: The notion of the five Platonic solids and the notion of the Harmony of the Spheres (Vlastos, 1975; Wilson, 1978). Thus, the orbits of the planets are ordered according to a specific arrangement of the five Platonic solids, while the motion of the planets produces a cosmic celestial polyphonic cantata. These normative scientific values are encompassed within the Keplerian paradigm and play an instructive role for the formation of the novel astronomical theory, so that old material is used and mixed with the new, in order for the astronomical breakthrough to be accomplished.The transformations of the planetary positions depend on a multiply connected symmetric and harmonic orderings that underlie the Cosmos these astronomical paradigms are referring to, while, in Kepler's paradigm, his three eponymous laws are used, together with the function of the five Platonic solids and the function of the Harmony of the Spheres, that is according to unifying harmonic meta-principles (Kepler, 1981; 1997).

Among the main achievements of the Keplerian paradigm belongs the determination of the orbit of Ceres by Gauss, where a planetary orbit between Mars and Jupiter should exist, based on a dissonance between the angular speeds of these two planets, evidenced by the smallest deviation perceptible. 


\section{THE COPERNICAN PARADIGM}

Copernicus and Rosen (1992) introduces his own paradigm, which is of heliocentric nature, but also rests upon the astronomical doctrines of the past, that is it heavily uses the theoretical premises and the mathematical structure of the Ptolemaic paradigm. Copernicus writes about the revolutions of the celestial bodies, placing the planets within a Cosmos, where the Sun serves as its geometric, not physical, center, thus moving the Earth from its stillness and at the same time, expanding the universe towards greater dimensions, by moving the sphere of the fixed stars far away, in order to predict the vanishing parallax of the fixed stars.

At the same time, Copernicus uses the whole of the conceptual framework and the mathematical machinery of the Ptolemaic paradigm, abandoning only the concept of the equant, an act of scientific revolution which caused the admiration of his fellow astronomers and polymaths, the ones who could understand deeply his astronomical theory (Kuhn, 1957). The abandoning of the notion of the equant refers to a deeply problematic feature of the Ptolemaic astronomical model, since it is being used as an auxiliary measure in order to justify the celestial path of the planets, especially for the case of Mercury, the planet with a most eccentric elliptical motion.

The only significant deviation from the Ptolemaic paradigm is the assumption and the theoretical embracement of the Heliocentric paradigm, as already stated within the work of Aristarchus of Samos, which is surely a great achievement of first order (Theodossiou et al., 2002). On the other hand, all the information and the tools for managing both the theoretical aspects of this astronomical theory, as well as for extrapolating the planetary positions on their orbits, that is the handling of the empirical data, is of the same complexity as the one which characterizes the Ptolemaic paradigm, to which we shall refer in the forthcoming paragraph. If we could loosely describe these complexities as the moods of the paradigms towards the physical Universe, we could state the opinion that the mood of the Copernican paradigm is almost the same as the one of the Ptolemaic paradigm, except from the crucial fact that the Heliocentric doctrine becomes the prevalent one, while the Geocentric doctrine gets for ever abandoned within the overall history of Astronomy.

\section{THE PTOLEMAIC PARADIGM}

Claudius Ptolemy introduces in his monumental work "The Mathematical Syntaxis", or "The Almagest", the epitome of the Hellenistic and Alexandrian astronomy, an astronomical paradigm which is going to remain as the only valid paradigm for many successive generations of astronomers and polymaths which followed after Ptolemy's era (Jones, 2010; Pedersen, 2011). Ptolemy introduces his astronomical and cosmological paradigm in a Euclidean fashion, starting with elaborate empirical statements, which he uses as axioms within his own astronomical model, just as Euclid used elaborate mathematical statements of obvious justification, serving as the basis of his Geometry (Mueller, 1969), that is as the axioms of his theory. All of Ptolemy's statements are self-evident, plausible and they stem from a grounded reality, from the empirical investigation of the heavenly phenomena, which lasted and accumulated for many centuries of observation and theoretical investigation and up to his age, as well as of the Physics of the terrestrial phenomena.

Ptolemy conceives the Aristotelian Universe, so his model is geocentric and geostatic, while all the planetary objects, the Sun and the Moon, revolve around the Earth within a prescribed ordering and according to Ptolemy's three rules of reproducing the planetary motions (Jones, 2010).

These rules save the appearances of the philosophical and theological doctrine, according to which the planetary motions have to reproduced by perfect circular motions, or by combinations of perfect planetary motions, in a manner which is consistent with the available empirical astronomical data. The retrograde motion of the planets, their changes in their magnitude and their phases as well, as observed during the course of the year, have to be justified and reproduced by combinations of cyclic motions, so that Ptolemy, already influenced by the Hellenic astronomical tradition, poses or introduces the notion of the eccentric planetary motion, by the notion of the epicycle and the deferent cycle and by the notion of the equant (Gingerich, 1981; Murschel, 1995). The last notion serves as a tool for the reproduction of the planetary motions and saves the phenomena, on the other hand its status is problematic, both epistemologically, as well as theologically, since the equant is just a mathematical point within the Ptolemaic Cosmos, without any reference to a particular physical body, to the physical body of the Earth.

The astronomers which followed the Ptolemaic model, belonging to the Byzantine, the Arabic and Islamic and the European civilization, devised numerous articulations and ad hoc modifications in order to eliminate any apparent conflict between this astronomical paradigm and the astronomical data at hand. Especially, a long tradition of Arabic and Islamic astronomers and polymaths (Saliba, 1994) tried to 
abandon the notion of the equant by the introduction of combinations of epicycles upon epicycles, which accurately reproduce the planetary orbits, although without being mathematically equivalent in a precise manner. The era of the Prolemaic paradigm, which can be considered to span the whole historical spectrum of the Hellenistic and Alexandrian epoch, that is from the very begin of the appearance of its main constituent elements, up to its precise formulation by the great Claudius Ptolemy, is also the era of the appearance of the first computing devices for the reproduction of the heavenly motions, as especially depicted within the elaborate construction and complex function of the Antikythera Mechanism, another epitome of the Hellenistic Astronomy, Technology, Philosophy and Physical science (Moussas, 2010; 2012; Freeth et al., 2006).

\section{APPLYING KUHN'S CRITERIA TO SUCCESSIVE ASTRONOMICAL PARADIGMS}

The successive astronomical paradigms we try to outline within the preceding work are totally characterized by the embodiment of their metaphysical values and doctrines, their own scientific vocabulary and language and by their own procedures, as well as the available instrumentation for justifying their existence within scientific terms. Each of these paradigms employs certain methods for the solution of the problems belonging to the sphere of its application, that is the astronomical and the cosmological one, as well as new areas of discourse to be investigated (Kuhn, 1997; 2000; 1996). The Ptolemaic paradigm already can be regarded as the epitome of the main scientific Hellenistic and Alexandrian tradition (Pedersen, 2011; Jones, 2010), but its further inner metamorphoses it has undergone in the hands of the Byzantine and the Arabic and Islamic astronomers shows directly its own internal dynamical evolution, as specific epistemological issues had to be resolved, just mentioning the case of the problematic issue of the equant, but also the confrontation with the even more accurate observational data had to be accomplished on even greater degree (Saliba, 1994; Kelley and Milone, 2005), that is in accordance with the fountain of the scientific truth, a fact which is never denied by Thomas Kuhn himself. The scientific theories, as expressed within these paradigms, should have, as a map, or even better, a conceptual mapping, a one-to-one correspondence with natural reality (Russo, 2004). This epistemological thesis is considered to hold for each one of the fathers of the studied paradigms within the present article, both of Ptolemy, Copernicus, Kepler (Cornford, 1922) and Newton, held the view that their astronomical and cosmological models described accurately the physical reality, both on the ontological, as well as the theological status. We may remark that there does not exist the justification of only one and single criterion for choosing among rival paradigms, but a certain combination of all the aforementioned normative rules, which are proposed by Thomas Kuhn, nor that this list of criteria is already exhaustive for evaluating one single paradigm, when compared to other rival paradigms belonging to the same era, or to different epochs of their appearance. Also, we have to stress the fact that Theology and Epistemology serve as necessary ingredients of these paradigms, as understood within the context of the historical and social environment of the scientific community, composed by succeeding generations of astronomers, polymaths and philosophers, up to Kepler's and even Newton's era. The natural realism of these paradigms is always combined by theological and philosophical considerations, so that the notion of truth is referring in a direct manner with the physical laws and these are always referring to the divine status quo of the Universe, the reflection of the Nous of the Demiourge of Cosmos (Burtt, 1954). Within these paradigms, the combined effort of deductive and inductive, as well as the abductive reasoning, is present. Their role for the structure and the function for the succeeding astronomical paradigms, from the transition from the Ptolemaic to the Copernican and the Keplerian paradigm, serves always as a regulating factor within the era of their normal existence, the era of the normal science, as applied to these, but also in the periods of the scientific revolutions, of the eras of interregnum between these paradigms and the introduction of the novel paradigm (Kuhn, 1957; 1996).

After having established a brief sketch of these successive astronomical paradigms in the preceding paragraphs, we can try to apply Kuhn's five criteria in a intra-theoretic manner, that is by the comparison of these paradigms with each other, as they appeared at the age of Kepler.

At the age of Kepler, just before the formulation of Kepler's three eponymous laws of the planetary motion, the Ptolemaic and the Copernican paradigm existed as two rival scientific visions of the then known Cosmos, of the Universe, as it was conceived by the scientific and astronomical community and the reigning philosophical and theological schools of their era.

These Paradigms can also be seen as "regression models", by fitting a formula to past data patterns, operating within an acceptable margin or error 
(Copernicus and Rosen, 1992; Pedersen, 2011). According to this view, we may encounter the Paradigm, that is one of its aspects, as a kind of a computational device, a specific kind of a Turing machine, which gives in its output the prediction or the retrodiction of the planetary positions based upon the available astronomical observational data, in the form of ephemeredes or tables (Kuhn, 1957). It is astonishing that among these two paradigms, at the era of the introduction of the Copernican paradigm, the first criterion, the criterion of accuracy, as imposed on the computational power, thus and on some aspects of the computational complexity of these paradigms, offered the same degree of justification. Both of these paradigms, whenever seen as computational devices, were exactly accurate in the reproduction of the paths of the planetary orbits. They reproduced numerically the prediction, or retrodiction, of the planetary paths within the same margin of error and even within the same realm of complexity, if referring to the amount of numerical computations which had to be undertaken by the proponents of these two paradigms in order to verify the position of the planets on the celestial sky. The calculation of the planetary motions within the realm of these paradigms did not offer any labor-saving techniques to the working astronomers, nor did they differ substantially among themselves within arithmetical accuracy. But, another aspect of simplicity refers to the economy introduced by the axioms of the theory and especially the theoretical conception of the motions of the planets. Here, we encounter a greater simplicity within the Copernican paradigm, since the Ptolemaic paradigm requires the arithmetical evaluation of a larger set of astronomical parameters. Thus, according to this feature of the simplicity criterion, the Copernican paradigm seems to offer a greater degree of parsimony, when compared to its rival Ptolemaic paradigm.

The internal consistency of each of these paradigms, that is of the Ptolemaic and the Copernican paradigm, this is a criterion which seems to be satisfied for both cases, since these do not seem to include any logical errors, or produce fallacies, although they are both referring to a completely different notion of Cosmos. They are, within their distinct formulations, both self-contained and selfconsistent, as a physical theory can ever be.

The other face of Kuhn's criterion, the external consistency, seems also to be satisfied, but also to be violated at the same time, according to the view we choose to study among these two astronomical paradigms, since the calculations performed within these two paradigms can already be used within the framework of Music theory, that is within the doctrine of the Music of the Spheres, as well as within the conceptual structure of Astrology. We mention these two disciplines, or levels of discourse, since from its very begin the science of Astronomy was deeply interlinked and connected to the realms of Astrology, Music theory, Alchemy and Medicine. This can be already seen in the work of the great founder of an axiomatically stated astronomical theory in the service of the Aristotelian cosmological paradigm, that is the Cosmos of Aristotle and in the work of Claudius Ptolemy. Ptolemy produced both the Mathematical Syntaxis and the Tetrabiblos, but he was also an important theorist in Music, as well. On the other hand, the geocentric and geostatic Ptolemaic paradigm could account for the most natural explanation of many terrestrial phenomena, all of them encountered within the realm of the Aristotelian physics, such as the fall of the heavy objects towards their natural place, the unmovable Earth, or the function of the water-pumps and the absence of cyclonic winds. The Copernican Earth, which moves freely in space and also rotates around its axis, cannot account for the explanation of these phenomena, so that the scientific and philosophic community had to wait for the advent of the Keplerian paradigm (Voelkel, 1999), which introduces in the most natural manner the concept of the gravitational force, as it stems from the Sun, the center of Cosmos and influences the planets, keeping them on their orbits, which share prescribed characteristics, according to Kepler's three Laws of planetary motion and furthermore the colossal Newtonian synthesis, which can account and explain these terrestrial phenomena, in terms of the Mechanistic philosophy of Nature.

On the other hand, the Copernican paradigm seems to offer a broader scope than the Ptolemaic paradigm, since it seems to enclose the totality of the celestial phenomena, a characteristic which shall be inherited in the Keplerian paradigm.

The unification offered by the Copernican paradigm is outstanding, if compared with the Ptolemaic paradigm. In the Ptolemaic Cosmos the Universe was divided within the two spheres of natural existence, the sublunar region and the region of the heavenly bodies, while these two distinct spheres were composed by different substances and obeyed different physical laws. While Ptolemy's Universe was divided, Copernicus offers the face of the Universe which is a united Universe, since a moving Earth is an Earth requiring the status of a planet and which does not function as the center of the 
Universe, makes this distinction useless and meaningless. This unity can be further understood by the fact that the quinta essentia abided by different laws than the terrestrial sphere, the sublunar region of the Universe consisted by the elements of fire, water, earth and air, while the celestial realm is composed of the fifth essence, the aether.

On the other hand, the fruitfulness of these two paradigms does not seem to be different, since both the Ptolemaic paradigm, as well as the Copernican paradigm, are considered to be built in order to justify and to explain the same set of the celestial phenomena.

The great rupture within the realm of Astronomy comes with the advent of the Keplerian paradigm, which can be both considered as an astronomical paradigm, as well as a cosmological paradigm, but also a paradigm for the science of Mechanics, the "natural philosophy of physics", as Newton states it explicitly in his most famous work in Physics, the Principia.

Kepler acknowledges the fact that the Ptolemaic, the Copernican and the Tychonic model, a clever mixture of the Ptolemaic and the Copernican model, are for all practical purposes equivalent within a hair's breath (Frisch, 1858-1872). Moreover, at that time, there was no way to determine the true organization and dynamics based on the discrepancies between the computational aspect, or computational power, as found between these Paradigms. Thus, Kepler has to begin his life-long struggle both in the corpus of the available astronomical data, the empirical data acquiring the best quality within the European scientific history, as obtained by the work of another giant of Astronomy, (Brahe, 1602), as well as with the available theoretical and mathematical machinery existing in his age.

As seen in an overall fashion, Kepler justifies even more the criterion of accuracy, since it reproduces in a most systematic and pragmatic fashion the description of the planetary orbits, it surpasses both the limit of acceptable error of the Ptolemaic, as well as of the Copernican paradigm.

Kepler's paradigm is also more consistent, both internally, as well as externally, than the aforementioned paradigms and for this reason we can mention two concrete examples. The first example is taken from Kepler's own remark, that the Ptolemaic orbit of the planet Mars should acquire a pretzel-like shape, something which cannot happen in reality. The second example refers to the fact that Kepler's own theoretical considerations are ultimately interlinked with the realms of Music theory and of Astrology, since they offer a most accurate account for the motion of the planetary objects, all that is needed within the discourse of these two branches of theoretical consideration and social discourse.

The criterion of broad scope holds also in favor for the Keplerian paradigm, since it includes the description of all celestial motions, for example the motions of the satellites of Jupiter, as discovered by Galileo and as studied by the next generation of astronomers and physicists. Kepler encompasses all the possible motions in his conception of Cosmos, as long as they obey his three eponymous laws, whether they refer to Jupiter's satellites, or the discovery of the orbit of another novel celestial body, that is the orbit of Ceres by the Prince of Mathematics, the most famous Carl Gauss.

The criterion of simplicity refers also to the Keplerian paradigm, in many aspects. Surely, it justifies the criterion of simplicity, as it has already been mentioned for the case of the Copernican paradigm. Also, since the Keplerian paradigm is a deep extension of the Copernican paradigm, it satisfies also other aspects of the complexity measure which could be attributed to the criterion of simplicity. The most obvious feature is the most elegant description of the planetary orbits in terms of ellipses, which can be understood as deferent cycles with the addition of an enormous number of systems of epicycles upon epicycles. The other feature refers to the fact that the eccentricities of the elliptical orbits of the celestial bodies, which could take any numerical value, as long as they belonged in an elliptical orbit attributing the same aphelion and the same perihelion point, obey Kepler's Harmonic law, so their values are further restricted by this physical requirement.

From all the above mentioned, we can understand that the fruitfulness of the Keplerian paradigm is richer than the one of the Ptolemaic and the Copernican paradigm and that this fruitfulness, as seen within the dynamical concept of a Universe, which obeys simple, dynamical Laws, is completely inherited within Newton's paradigm, so that everything which can be stated about Newton's paradigm, can also be stated about Kepler's paradigm, with Kepler as one of the giants, on whose shoulders Newton stood. While the fruitfulness of the Ptolemaic and the Copernican paradigm do not seem to vary considerably, the fruitfulness of the Keplerian paradigm seems to serve as an origin for some of the pillars of the Newtonian paradigm, but on the other hand some of its aspects, inherited within the philosophical thought of the great Leibniz, seem to be lost in the post-Newtonian tradition of the Mechanistic philosophy. 


\section{DISCUSSION}

After having studied these successive astronomical paradigms, we may remark that one pragmatic manner for choosing a paradigm may not exist within the realm of certain epochs of the science of Astronomy, where many rival theories may coexist and claim the justification of the totality of the observed physical phenomena (Kordig, 1971). At the end, after the coming of a novel scientific era, the available scientific instrumentation and the innovations of technology, which provide a more detailed aspect of reality, push forward into the consciousness of the scientific community the paradigm which stays in accordance with the empirical evidence (Kuhn, 1993), as it happened with the Keplerian paradigm, at the time it surpassed by far both the Ptolemaic, as well as the Copernican paradigm, but also the Tychonic paradigm.

There is no failure of method, or of scientific explanation, between these paradigms, all of them follow strictly the scientific method, an offering of the Hellenic and Hellenistic Astronomy to the forthcoming great civilizations (Russo, 2004). We remark that between these competing paradigms there exists the differences of the non-empirical, or even better, the trans-empirical elements. The astronomers look up in the sky and observe, but they do see different kinds of "realities". Even Kepler saw a reality composed by the intercalation of the five platonic solids in each other, his vision of Cosmos, as stated within his first great work, the Mysterium Cosmographicum, remained according to him the only and true depicture of the underlying fabric of physical reality, making this theory one of the most beautiful, but also wrong, depictions of reality (Kepler, 1981 ; 1997). Kepler insisted in this primary vision of Cosmos, until the end of his life and his scientific work both encompassed the Cosmic Mystery of a Neoplatonic Cosmos (Hopkins, 2001), by obeying his three eponymous laws of the planetary motions.

We may encounter the Paradigm, that is one of its aspects, beyond its syntactic and semantic structure and as a kind of computational device, a specific kind of a Turing machine, which gives in its output the prediction or the retrodiction of the planetary positions based upon the available astronomical observational data, in the form of astronomical ephemeredes or tables.

According to this aspect, we remark that the Ptolemaic and the Copernican paradigm offer the same computational complexity for the determination of the planetary orbits and the computational complexity of Kepler's paradigm does not differ so much from the one of these preceding paradigms, it offers a most detailed framework for the computation of the orbital elements of each and every existing or newly-discovered celestial body within the Keplerian, or the Newtonian Universe. Thus, the complexity of its abstract theoretical structure is far more advanced, it contains more information and offers simpler ways for determining the physical underlying structure of the celestial objects, as well as the Keplerian elements of their particular motions.

Furthermore, the theoretical complexity of Kepler's paradigm is richer, it contains more information than the Ptolemaic and the Copernican paradigm, since it introduces within its conceptual framework a host of novel notions and ideas, such as the notion of dynamical physical laws, the notion of the gravitational force, in its primary form, as well as the notions of Infinitesimal Calculus, that is of Integration, in his own Universe. These theoretical structures succeed by far the static geometrical structure, as offered in the Ptolemaic and the Copernican paradigm, both in their explanatory power and within the re-formulation of the physical reality according to the existence of physical forces, as described by simple, accurate mathematical laws.

Copernicus, as well as Kepler become the leaders of their own astronomical paradigms, guided both by their theological and metaphysical convictions, as well as the confrontation with the available empirical observational data, without paying much attention to the strict evaluation of the already proposed paradigmatic criteria (Kuhn, 1957; Stephenson, 1994). The great Newton seems also to follow the same lines. But, all the forthcoming generations of scholars and physicists seem to apply especially all of Kuhn's proposals for a sound and firm scientific theory, in one way or another, as we can observe within the spread of the postNewtonian paradigm along the circles of the astronomers and the philosophers of the European civilization (Dijksterhuis, 1986).

This breakthrough within the realm of Astronomy, as well as within the realm of Physics, shall always stand as a landmark of physical explanation and as one of the pillars of the forthcoming Newtonian paradigm and also as one important ingredient of the Worldview of another giant of Astronomy, Physics, Mathematics and Philosophy, in the European civilization, the most famous Gottfried Leibniz (Dijksterhuis, 1986).

Many aspects of Kepler's own vision of the Universe, together with the newly introduced measures of complexity of the theoretical framework of his planetary theory, shall cross-fertilize other aspects of the European tradition of viewing the Cosmos as an organic whole, dictated by concrete physical laws, obeying symmetries 
and harmonies, not only in the study of the celestial motions, but also in the study of other aspects of physical reality, such as the biological phenomena. This richness is another measure of Kepler's own vision and a landmark of the ingenuity of one of the most important spiritual figures within the history of European thought, who stays as equally placed among Copernicus and Ptolemy, Tycho Brahe and Isaac Newton, in the pantheon of the giants of Physics and Astronomy.

\section{ACKNOWLEDGMENT}

The researchers express their gratitude to the National Research Foundation Library.

\section{REFERENCES}

Ben-Menahem, A., 2009a. The Clockwork Universe. In: Historical Encyclope dia of Natural and Mathematical Sciences, Ben-Menahem A. (Ed.), Springer, Berlin Heidelberg, ISBN-10: 3540688315, pp: 911-1731.

Ben-Menahem, A., 2009b. Deep Principles-Complex Structures. In: Historical Encyclopedia of Natural and Mathematical Sciences, Ben-Menahem A. (Ed.), Springer, Berlin Heidelberg, ISBN-10: 3540688315, pp: 5081-5986.

Brahe, T., 1602. Tychonis Brahe Astronomiae Instauratae Mechanica (Smithsonian Institution Libraries.

Brotton, J., 2006. The Renaissance: A Very Short Introduction. 1st Edn., Oxford University Press, ISBN-10: 0191577553, pp: 160.

Burtt, E.A., 1954. The Metaphysical Foundations of Modern Physical Science. 2nd Edn., Doubleday, Garden City, N.Y., pp: 352.

Caspar, M., 1993. Kepler. 1st Edn., Courier Dover Publications, ISBN-10: 0486676056, pp: 441.

Cooper, G.M., 2011. Galen and astrology: A Mésalliance? Early Sci. Med., 16: 120-146. PMID: 21879603

Copernicus, N. and E. Rosen, 1992. On the Revolutions.1st Edn., Johns Hopkins University Press, Baltimore, ISBN-10: 0801845157, pp: 452.

Cornford, F.M.,1922. Mysticism and science in the pythagorean tradition. Classical Q., 16: 137-150.

Dijksterhuis, E.J., 1986. The Mechanization of the World Picture: Pythagoras to Newton. 1st Edn., Princeton Univ Pr, ISBN-10: 0691023964, pp: 552.

Dreyer, J.L.E., 1967. A History of Astronomy from Thales to Kepler. 2nd Edn., Dover Publications, New York, pp: 438.
Fisher, G., 2006. Marriage and Divorce of Astronomy and Astrology: A History of Astral Prediction from Antiquity to Newton. 1st Edn., Lulu.com, Morrisville, N.C., ISBN-10: 1411683269, pp: 232.

Freeth, T., Y. Bitsakis, X. Moussas, J. Seiradakis and A. Tselikas et al., 2006. Decoding the ancient Greek astronomical calculator known as the Antikythera Mechanism. Nature, 444: 587-591. DOI: 10.1038 /nature 05357

Frisch, C., 1858-1872. Ioannis Kepleri Astronomi Opera omnia. 1st Edn., Frankfurt a.M and Erlangen, Heyder and Zimmer,

Gingerich, O., 1981. Ptolemy Revisited-Reply to Newton, R.R. Q. J. Royal Astronomical Society, 22: 40-44.

Gutting, G., 1980. Paradigms and Revolutions: Appraisals and Applications of Thomas Kuhn's Philosophy of Science. 1st Edn., University of Notre Dame Press, ISBN-10: 0268015422, pp: 339.

Heidelberger, M., 1976. Some intertheoretic relations between ptolemean and Copernican astronomy. Erkenntnis, 10 : 323-336. DOI: 10.1007/BF00214727

Hopkins, J., 2001. Complete Philosophical and Theological Treaties of Nicholas of Cusa. Arthur J Banning Pr, ISBN-10: 093806049X, pp: 676.

Hudson, J., 1992. From Alchemy to Chemistry. The History of Chemistry. Springer US, pp: 35-46.

Jones, A., 2010. Ptolemy in Perspective: Use and Criticism of his Work from Antiquity to the Nineteenth Century. 1st Edn., Springer, Dordrecht, ISBN-10: 9048127874, pp: 229.

Kelley, D.H. and E.F. Milone, 2005. Observational Methods and Problems. In: Exploring Ancient Skies: An Encyclopedic Survey of Archaeoastronomy, Kelley, D.H. and E.F. Milone (Eds.)., Springer, New York, pp: 49-83.

Kepler, J., 1981. Mysterium cosmographicum: The secret of the universe. New York.

Kepler, J., 1997. Harmonice Mundi Libri V. In: The Harmony of the World, Aiton, E.J., A.M. Duncan and J.V. Field (Eds.), American Philosophical Society, Philadelphia, ISBN-10: 0871692090, pp: 549-549.

Kordig, C.R., 1971. The Theory-Ladeness of Observation. In: The Justification of Scientific Change, Kordig, C.R., (Ed.), Springer, Dordrecht, ISBN-10: 9027701814, pp: 1-33. 
Kuhn, T.S., 1957. The Copernican Revolution: Planetary Astronomy in the Development of Western Thought. 1st Edn., Harvard University Press, Cambridge, MA, ISBN-10: 0674171039, pp: 297.

Kuhn, T.S., 1974. Second Thoughts on Paradigms. In: The Structure of Scientific Theories, Suppe, F., (Ed.), University of Illinois Press, Champaign, ISBN-10: 0252006348, pp: 459-82.

Kuhn, T.S., 1977. The Essential Tension: Selected Studies in Scientific Tradition and Change. 1st Edn., University of Chicago Press, Chicago, ISBN-10: 0226458067, pp: 366.

Kuhn, T.S., 1993. Afterwords. In: World Changes: Thomas Kuhn and the Nature of Science, Horwich, P., Ed., University of Pittsburgh Press, Pittsburgh, Pa., ISBN-10: 0822960540, pp: 311-341.

Kuhn, T.S., 1996. The Structure of Scientific Revolutions. 3rd Edn., University of Chicago Press, Chicago, IL, ISBN-10: 0226458083, pp: 212.

Kuhn, T.S., 2000. The Road Since Structure: Philosophical Essays, 1970-1993, with an Autobiographical Interview. 1st Edn., University of Chicago Press, Chicago, ISBN-10: 0226457982, pp: 335.

Kutash, E., 2011. Ten Gifts of the Demiurge: Proclus on Plato's Timaeus. 1st Edn., Bloomsbury Academic, ISBN-10: 0715638548, pp: 309.

Laiou, A.E., 1992. Byzantium: A World Civilization. 1st Edn., Dumbarton Oaks, Washington, D.C., ISBN10: 0884022005, pp: 162.

Laudan, L., 1986. Scientific change: Philosophical models and historical research. Synthese, 69: 141223. DOI: $10.1007 / \mathrm{BF} 00413981$

Llyod, G.E.R., 1973. Greek Science after Aristotle. 1st Edn., W W Norton and Company Incorporated, New York, ISBN-10: 0393007804, pp: 189.

Margolis, H., 1993. Paradigms and barriers: How habits of mind govern scientific beliefs. 1st Edn., University of Chicago Press, Chicago u.a., ISBN10: 0226505235, pp: 267.

Marshall, P., 2009. The magic circle of Rudolf II: alchemy and astrology in Renaissance Prague. 1st Edn., Bloomsbury Publishing, ISBN-10: 0802718574, pp: 288.

Meri, J.W., 2004. Medieval Islamic Civilisation. 1st Edn., Routledge, New York.

Moussas, X., 2010. The Antikythera Mechanism. In: Adapting Historical Knowledge Production to the Classroom, Section, A. (Ed.(., ISBN-10: 978-946091-349-5, pp: 113-128.

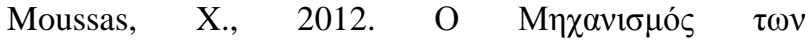

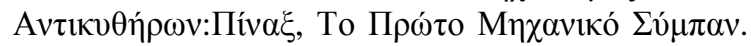
The Antikythera Mechanism: Pinax, The First Mechanical Universe.

Mueller, I., 1969. Euclid's elements and the axiomatic method. Brit. J. Philosophy Sci., 20: 289-309.

Murschel, A., 1995. The structure and function of ptolemy's physical hypotheses of planetary motion. J. History Astronomy, 26: 33-61.

Pacholczyk, J., 1996. Music and Astronomy in the Muslim World. Leonardo, 29: 145-150.

Pedersen, O., 2011. A Survey of the Almagest. 1st Edn., Springer, New York,

Robertson, R., 2009. Indra's Net: alchemy and chaos theory as models for transformation.

Russo, L., 2004. The Forgotten Revolution: How Science Was Born in $300 \mathrm{BC}$ and Why it Had to be Reborn. 1st Edn., Springer Berlin Heidelberg, ISBN-10: 3540200681, pp: 487.

Saliba, G., 1994, A History of Arabic Astronomy: Planetary Theories During the Golden Age of Islam. 1st Edn., University Press, New York,

Schroedinger, E., 1996. Nature and the Greeks and Science and Humanism. 1st Edn., Cambridge University Press, pp: 160.

Stephenson, B., 1994. Kepler's Physical Astronomy. 1st Edn., Princeton University Press, Princeton, N.J., ISBN-10: 0691036527, pp: 216.

Taton, R. and C. Wilson, 1989. Planetary Astronomy from the Renaissance to the Rise of Astrophysics, Part A, Tycho Brahe to Newton. 1st Edn., Cambridge University Press, Cambridge, ISBN10: 0521242541, pp: 304.

Theodossiou, E., E. Danezis, V. Manimanis and E.M. Kalyva, 2002. From pythagoreans to kepler: The dispute between the geocentric and the heliocentric systems. J. Astronomical History Heritage, 5: 89-98.

Vlastos, G., 1975. Plato's Universe. 1st Edn., Clarendon Press, Oxford, ISBN-10: 0198245386, pp: 130.

Voelkel, J.R., 1999. Johannes Kepler and the New Astronomy. 1st Edn., Oxford University Press, New York, ISBN-10: 0195116801, pp: 141.

Wilson, C.H., 1978. Harmonies and the Exactitude of Kepler's Third Law. In: Science and History: Studies in Honor of Edward Rosen, Hilfstein, E. and F.D. Grande, (Eds.), Ossolineum, pp: 235-59. 